\title{
FUNDAMENTALS OF ART WITH SPECIAL REFERENCE TO LINE AND SHAPE
}

\author{
Dr. Kumkum Bharadwaj $1 ه$ \\ Professor and Head, Drawing and Painting Department, Maharani Lakshmi Bai Government Girls College \\ Indore Madhya Pradesh, India
}

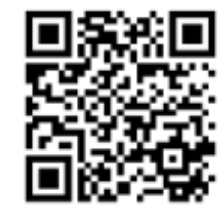

\section{ABSTRACT}

The most prominent and artistic art is Painting. If an artist is willing to create a painting, then it requires that cooperation of some of the main essential basics, medium or elements of painting, which can be followed to create an excellent painting.

Keywords: Fundamentals, Special, Line and Shape

Received 15 December 2020

Accepted 25 February 2021

Published 25 February 2021

CorrespondingAuthor

Dr. Kumkum Bharadwaj,

kumkbh@gmail.com

DOI

10.29121/shodhkosh.v2.i1(SE).2021. 16

Funding: This research received no specific grant from any funding agency in the public, commercial, or not-for-profit sectors.

Copyright: (C) 2021 The Author(s). This is an open access article distributed under the terms of the Creative Commons Attribution License, which permits unrestricted use, distribution, and reproduction in any medium, provided the original author and source are credited.

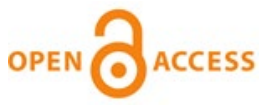

\section{INTRODUCTION}

Art creation has been mostly personal. Working from centuries, Art creator as a painter has established some concepts of line, form, colors, texture, space, value, perspective, and various mediums. These seven most common elements. Elements of art are the features of art style that help the artist to communicate artistic views because art is a medium of communication. Art creation of a creator is completely personal, and expression are different but there are some fundamental elements are natural and available in all paintings. On the basis of these elements so much attention should always be followed at the time of creating a painting. These elements are not like mathematical formulas, but they are most flexible and lively.

Line: Line is the most fundamental element or one of the basic and expressionative element of any painting no matter the medium. Line refers to mark which spans between two points. Line is a mark made using a drawing tool or brush. With the indication of line, many imaginations come alive and take the viewer in to imaginative world. Bhargava (n.d.) By analyzing types of lines used in various works of art to help the audience understand how artist use line to convey movement and mood. Lines are created when one point connects to another. They are inherent in all visual arts from painting to Sculpture even performing arts also.

In the painting lines are made when an artist draw and paint on paper, canvas and board or any surface. In Indian art lines are most powerful and expressive. According to Griffith - "The expression of Indian painting is praised for its expression, the basics of which is the drawing of Indian painting." Udatta Bhawna ek vishleshan (2005) If we see the history of Indian painting, from prehistoric to modern painting, lines are main part of communication and expression. Pre- historic paintings are based only lines. 
Some powerful ideas can help to understand that how a painting expressed through use of lines and lines contribute an important role to prepare a painting. Some ideas are here to help to understand the element of line:

- An artist and an audience also should have knowledge of different types of lines, it means the vocabulary of lines should be rich to create a painting.

- Artist and art praiser also should have practice the style of lines, under the style they should compare and contrast of lines.

- More studying the portraiture art so that different types of lines learn and understand.

- In a painting an artist uses both smooth and rough lines at a time so audience also should have knowledge smooth and rough lines

- An artist should Focus online work, emphasize the emotions with the help of lines.

There are an endless number of lines that use in painting, and it is a starting point for creation of painting. There are many types of lines; thick, thin, horizontal, vertical, zigzag, diagonal, curly, curved, spiral etc. Some lines in painting are invisible that cannot see the dark mark of the line, but they are there in painting, shown in the way the artist manages the figures, objects and other aspects of painting. Straight lines and curved lines are the starting of a painting after that an artist give a shape to full painting.

\section{Utilization of lines in a painting}

An artist uses different types of lines in a painting and these lines use to different ways and purpose by an artist. When deeply analysis in this matter some aspects obtained from paintings, these are following purpose to uses lines:

- Lines are used for sketching, gesture and drawing so that artist can create an image with just online.

- Lines define objects, to outline shapes draw a composition, layout before painting on art panel

- All shading techniques are based on the use of lines whether straight lines are used or curved lines, or cross hatching etc. They are all using some types of lines drawing as longer strokes or variation of strokes to show depth, tone, and texture also. Means shape, tone, texture, value all elements of art are dependent on lines.

- In addition to shading techniques lines can be used alone as an element to add depth in a painting. Manipulating a line; blending it, changing its thickness, to achieve an illusion of three-dimensional objects and space. Etching, engraving, and print media are excellent example of line technique.

- Lines are the greatest supporting tool. Lines drawing using grid method and constructing forms and objects with working linear perspective.

- An artist uses the lines to decorate with the help of simple pattern of lines.

- Abstract Painting is a common use of lines Op art is a good example of abstract Painting 


\section{TYPES OF LINE DRAWING}

- Freehand drawing

- Memory drawing

- Representational drawing

- Mechanical drawing

- Contour drawing

- Gesture drawing

- Nature drawing

- $\quad$ Object drawing

- Knowing the tools and Materials: Expressive aspects of lines -Prehistoric lines, Ajanta, Apbhransha, Mughal and Rajasthani lines have great possibilities in painting sector. But we can also produce different linear effects by the difference of manual movement method

- Drawing the wrist with the motion method of fingers only by stability it on the ground.

- By lifting the wrist from the ground and resting elbow to draw.

- To draw the line by keeping the wrist and elbow apart from the ground.

\section{Emotional qualities of lines}

Lines have great feelings, by changing the way or shading can change the meaning and message of a painting.

- Horizontal lines are quiet, calm and show distance. Horizontal lines show that there is a space and distance in a painting.

- Vertical lines give an impression of height, balance, support, and strength.

- Curved lines are very special. They are very comforting comparing with straight lines elegance and graceful. If an artist wants to decorate some object, should use curve lines for decoration.

- Zig-Zag lines create all kind of energy. This type of lines suggests an anxiety and confusion also. Zig-Zag parallel lines have speed and movement, but slanted lines are also full of energy and support movement and action in a painting. They can also emphasize and lead viewers eyes to specific spot in a painting.

- Diagonal lines that straight in any direction except vertical or horizontal lines.

An artist uses different varieties of lines like long, short, thin, thick, smooth, rough, doted, dashed, continuous and implied lines in a painting and changing the direction and degree of curve can explain subject, feelings. Lines have energy and rhythm. Rhythm is fundamental to organic growth so that lines are main element of any composition. 
Form: The term form means several different things in art. Form is one of the seven elements of art and three-dimensional single object in space. Form is also used to describe the physical nature of a painting. The importance of form for a painting is the first part - "Roop binds many in to one. In the absence of forms, it is not possible to experience 'one', one in which many lives are contained in form itself, balance and dialogue are born." Prasad (1996) Form for painting is an essential element. The marking form is done with the help of colours and lines from the paint brush. The main source of the form is point which has all the potential of the form and also the capacity of form creation. An artist looks at all forms with his / her own emotions and creates an image of his / her interest." When a shape is repeated and again, rhythm is produced in it." Saxena (2002) Form and structure refer to use of visual elements to convey three dimensional objects on a flat surface. This could be done through methods such as shading, contrast or contour drawing.

Most subject can be simplified down to basic forms of sphere, boxes, and cylinders. Understanding the forms and structures which make up painting subject, it will allow to paint more convincing depictions. The Idea of form is very general but also very important. Artists who learn to recognise form see beyond the two D paper or screen. They were able to create objects and that look realistic because forms look jubilant and lively.

As an element of painting form that is three dimensional and enclose volume, having length, width, and height, versus shape which two dimensional or flat. The forms can be divided in to two major groups:

1) Equilateral- In which the shapes of the form are fixed and are basically based on geometric shapes. Circle, glass, cube etc. These shapes have less intellectual creativity and monotonous in nature. Geometric forms are most often found in architecture.

2) Asymmetrical- These shapes are free from geometrical bonds and the shapes are not fixed but change in physical form, the forms can be divided into two parts- first tangible form second intangible. Both direct and indirect forms are easily expressed. This type of forms is difficult to balance but more interesting.

Indian scholars pondered a lot about the form design and detailed it in three ways:

1) Subtle forms: It consists of those forms which are not even in nature or geometric forms etc.

2) Natural forms: some forms are created by God are so beautiful and natural forms. Natural forms are part of nature and God made human being has no hand.

3) Decorative forms: decorative forms created by a new expression, new motion and special attraction to natural forms, artist is able to become ornamental when he creates the form, but it is the birth of nature.

All forms are originally geometric only, but artist see by his / her imagination and perception. Renowned artist Pablo Picasso has shaped the objects and human beings also in geometric forms. Cubism is the excellent example of this type of art forms.

In painting the illusion of three-dimensional form is conveyed through the use of lighting, shadow and rendering of value and tone. Form is defined by the outer 
contour of an object, which is how the artist firstly observe it and start to make sense of it but light, shadow, and value helpful to give shape an object and context in space so that audience can identify the exact form. Historically, if we observed, painting has progressed from flat representation of form and space to a three-dimensional, representation of form and space to abstraction.

Effects of Form: Form create different types of effects in the painting. The artist creates his/ her subjective effect by marking the form in different types of forms. According to lines forms also have own effects, these are the cause of visual and formal weight. The effects of the form can be following:

- Rectangular: strength, stability, and unity

- Triangular: permanence, security, development, or evolution.

- Reverse Triangular: Entanglement and disturbance.

- Inverted triangular: involvement and unrest.

- Ovals: Grace, charm, perpetuation, and creativeness.

- Circular: Fullness, charm, motion, immensity grandeur, perfection, speed, and equality.

- Elliptical: Beauty, continuity, and creativity

An artist continues to explore the many possibilities of form and new dimensions are presents the audience. Ajanta, Apbhransha, Rajasthani, and Mughal paintings have present different types of original forms in Indian painting, these are the fundamental of painting in Indian art.

\section{REFERENCES}

Prasad, P. (1996). Chaumasa. Lokranjan, 18-20.

Saxena, S. N. (2002). Visual Art And Vision, 46.

Bhargava, S. (2019). Saundryabodh Aur Lalit Kalayen, 69.

Udatta Bhawna ek vishleshan (2005). Jaipur : Premsagar Rajasthan Hindi Granth Academy. 